\title{
281 MEASURING IMMUNE CHECKPOINT INHIBITOR EFFICACY USING PRIMARY PATIENT-DERIVED 3D SPHEROIDS
}

Kathryn Appleton*, Katy Lassahn, Ashley Elrod, Tessa DesRochers. KIYATEC, Inc., Greenville, SC, USA

Background Cancerous cells can utilize immune checkpoints to escape T-cell-mediated cytotoxicity. Agents that target PD-1, PD-L1 and CTLA4 are collectively deemed immune checkpoint inhibitors (ICIs), and many have been approved for treatment of non-small cell lung cancer (NSCLC) and melanoma. Unfortunately, many patients do not respond to these therapies and often experience disease progression. Immunohistochemistry assays to predict response to ICIs have been inconsistent in their readouts and often patients with low expression levels respond to ICIs. Understanding the determinants of ICI response in individual patients is critical for improving the clinical success of this drug class. Using patientderived spheroids from NSCLC and melanoma primary tissue, we developed a multi-plexed assay for detecting ICI efficacy.

Methods Nine NSCLC and 11 melanoma primary tumor samples were dissociated to single cells, classified for immune checkpoint expression and cell content by flow cytometry, and seeded for spheroid formation. Spheroids were treated with pembrolizumab, nivolumab, atezolizumab, ipilimumab or durvalumab across a range of concentrations and monitored for cytotoxicity at 24-hours and viability at 72 -hours by multiplexing CellTox ${ }^{\mathrm{TM}}$ Green Cytotoxicity Assay and CellTiterGlo ${ }^{\circledR}$ 3D Cell Viability Assay. IFN $\gamma$ and granzyme B secretion was assessed using Luminex technology. ICI response was evaluated by determining the concentration-response relationship for all three read-outs.

Results Increased IFN $\gamma$ and granzyme B were detected for every ICI in one or more patient samples. ICI-induced IFN $\gamma$ secretion inversely correlated with PD-1+ immune cells. Durvalumab was significantly more cytotoxic for both NSCLC and melanoma spheroids compared to the other ICIs and significantly reduced spheroid viability with mean spheroid survival decreasing to $19.5 \%$ for NSCLC and $58.2 \%$ for melanoma. We evaluated if there was an association between durvalumab response and cell composition and found that percent spheroid survival significantly correlated with CD8 + Tcells for both NSCLC $(\mathrm{r}=-0.7920, \mathrm{p}=0.0191)$ and melanoma $(r=-0.6918, p=0.0390)$. Furthermore, CD8 + T-cells correlated with durvalumab-induced granzyme $\mathrm{B}$ secretion for NSCLC $(\mathrm{r}=-0.7645, \quad \mathrm{p}=0.0271) \quad$ and melanoma $(\mathrm{r}=-0.7419$, $\mathrm{p}=0.0221)$.

Conclusions In this study we show ICI-specific increases in immune-related analytes in a concentration-dependent manner for NSCLC and melanoma patient-derived spheroids. We detected spheroid cytotoxicity following short term ICI treatment which closely mirrored decreased spheroid viability at a later timepoint. Finally, we can decipher response mechanisms as exemplified by durvalumab-induced granzyme B secretion correlating with the presence of $\mathrm{CD} 8+$ T-cells which results in reduced spheroid viability for both tested cancer indications.

http://dx.doi.org/10.1136/jitc-2021-SITC2021.281 